\title{
Uterine torsion in pregnancy, expect the unexpected: case report
}

\author{
Huda Nabihah Mokhtar*, Sumayyah Mohammed Faridz, Rohana Ismail, \\ Nasuha Yaacob, Roziana Ramli
}

Department of Obstetrics and Gynecology, Hospital Sultanah Nur Zahirah, Kuala Terengganu, Malaysia

Received: 18 May 2020

Accepted: 29 June 2020

\section{*Correspondence:}

Dr. Huda Nabihah Mokhtar,

E-mail: drhudamokhtar@gmail.com

Copyright: (C) the author(s), publisher and licensee Medip Academy. This is an open-access article distributed under the terms of the Creative Commons Attribution Non-Commercial License, which permits unrestricted non-commercial use, distribution, and reproduction in any medium, provided the original work is properly cited.

\begin{abstract}
Uterine torsion in pregnancy is a rare condition, but potentially life-threatening. The non-specific clinical features make preoperative diagnosis difficult and most cases are discovered during caesarean deliveries done for other obstetric indications. Authors present 2 cases of uterine torsion with different clinical presentation and outcome. Case 1 was a G3P2 at 36 weeks with twin pregnancy presented in active labour with a prolapsed cord. A 180 degrees uterine torsion was discovered only after delivery of the foetuses, resulting in inadvertent posterior uterine wall incision. Case 2 was a G6P5 at 35 weeks who presented with an acute abdomen and went into shock. At laparotomy, a 360 degrees uterine torsion was discovered complicated with placental abruption, causing a fresh stillborn baby. These cases highlight uterine torsion as a rare but important diagnosis in obstetrics, which could be associated with potentially devastating outcome.
\end{abstract}

Keywords: Inadvertent posterior uterine wall incision, Uterine torsion during pregnancy, 180 degrees uterine torsion, 360 degrees uterine torsion

\section{INTRODUCTION}

Uterine torsion is a common problem in veterinary medicine but considered rare in human and has been referred to as an 'obstetrician's once in a lifetime diagnosis'. Although rare, it is associated with significant maternal and fetal morbidity and mortality. Torsion of less than 45 degrees is considered physiological, but rotation of the uterus of more than 45 degrees on its longitudinal axis is considered pathological. Most cases of uterine torsion reported in the literature were around 180 degrees and most often dextrorotatory, but torsion up to 720 degrees have been reported. ${ }^{1}$

\section{CASE REPORT}

\section{Case 1}

A 33-year-old, G3P2 at 36 weeks pregnancy with monochorionic diamniotic twins presented in active labour. The lead twin was in cephalic presentation but not engaged. Vaginally, she is $4 \mathrm{~cm}$ dilated and a prolapsed pulsating cord was felt. Red alert was activated and the case was managed as per protocol for cord prolapse. The patient was immediately rushed to the operation theatre for a crash caesarean section. The abdomen was opened through Pfannensteil incision. The lower uterine segment was incised transversely without separating the uterovaginal fold to expedite delivery. Both twins were delivered easily with good Apgar score. After delivery, the uterus was exteriorized to facilitate surgical closure. To this surprise, the uterine incision was found to be inadvertently made at the posterior uterine wall due to a 180 degrees uterine torsion. Manual correction of the torted uterus was done before closing the uterine incision (Figure 1, Figure 2). The patient made an excellent postoperative recovery.

\section{Case 2}

A 40-year-old, G6P5 at 35 weeks was managed expectantly in obstetrics ward for placenta praevia type 
IV major. While in ward, she complained of sudden onset of severe abdominal pain, not typical of labour pain and not associated with vaginal bleeding. Clinically she was restless and was in shock, with low BP of $50 / 30 \mathrm{mmHg}$ and pulse rate of 107 beats per minute. Abdomen examination revealed a tensed and tender uterus with fetal bradycardia ( 60 beats per minute). Red alert was activated for maternal collapse with acute abdomen. Differential diagnosis includes ruptured uterus, concealed abruptio placenta or surgical cause e.g. perforated viscus. Decision for exploratory laparotomy was made after adequate resuscitation. Intraoperatively, the uterus was noted to be twisted 360 degrees at the cervico-uterine angle, obscuring the lower uterine segment. Manual repositioning of the uterus attempted but failed. The uterus was incised vertically, and unfortunately a fresh stillborn baby was delivered. Placental abruption was detected with $1000 \mathrm{mls}$ of blood clots. Interestingly, upon detorsion of the uterus to its normal position, the placenta was seen occupying the upper uterine segment, not 'placenta previa' as previously reported. The patient made a full recovery and discharged well.

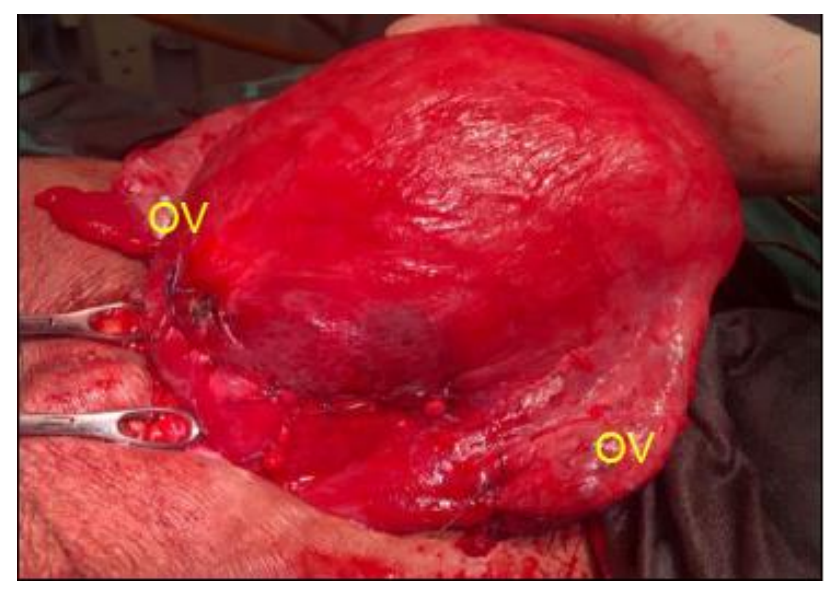

Figure 1: Sutured transverse caesarean incision at posterior uterus as evidenced by both ovaries (OV) located most anterior.

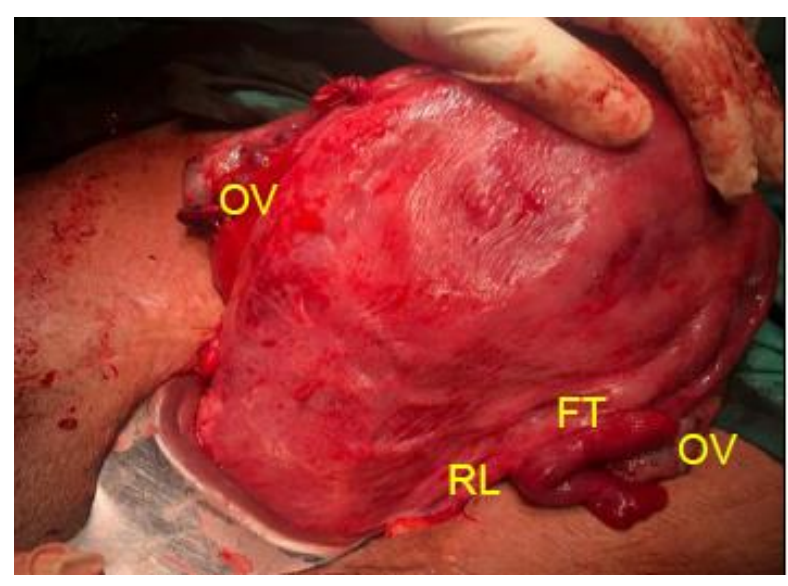

Figure 2: Anterior uterus as seen with left round ligament (RL) located most anterior, followed by left fallopian tube (FT) and left ovary (OV).

\section{DISCUSSION}

Uterus is an interesting organ, shaped like an upsidedown pear that sits on a hammock of muscles of the pelvic floor. Obvious asymmetry due to congenital or acquired deformities (e.g. uterine fibroids, uterine anomalies, pelvic adhesions etc.) could predispose to uterine torsion. In addition, structural weakness (developmental or acquired) and angulation in the isthmic region could also lead to torsion. ${ }^{2}$ Pregnancy exaggerates the congenital and physiologic rotations and obliquities of the uterus, this explains why uterine torsion occurs more frequently in gravid uterus compared to non-gravid uterus. $^{1,3}$

The exact etiology of uterine torsion however, is not known and it is independent of maternal age, parity and gestational age. ${ }^{1}$ More than 60 years ago, Nesbitt and Corner analyzed 107 cases of uterine torsion in the world's literature and found the common causative factors of uterine torsion include uterine fibroids, uterine anomalies, pelvic adhesions and ovarian cysts. ${ }^{4}$ The remaining $30 \%$ of patients had no discoverable cause. Jensen et al, did a more comprehensive review analyzing 212 cases of uterine torsion and found association with abnormal fetal presentations (23\%), uterine fibroids $(21 \%)$, uterine malformations $(11 \%)$, pelvic adhesions $(7 \%)$ and other factors $(27 \%)$ including placental problems (abruption, previa) and overdistended uterus (polyhydramnios, twins) among other causes. ${ }^{1}$ In contrast with Nesbitt's series, there were no abnormalities found in $16 \%$ of cases. Interestingly, the most frequent abnormal foetal presentations described in Jensen's series were transverse lie (72\%). ${ }^{1}$ Other possible causes described by other authors include external cephalic version (ECV), maternal trauma and abdominal massage. ${ }^{5-7}$ Recent review however, found uterine torsion occurred within a typical pelvis and not associated with any pelvic pathology. ${ }^{8}$ In case 1 , multiple pregnancy could possibly be the triggering factor for uterine torsion but in case 2, no obvious pathology was found.

Uterine torsion is a potentially dangerous complication of pregnancy both to the mother and to the foetus. Nesbitt and Corner in 1956 stated that overall maternal mortality is around $13 \%$ and is directly proportional to the duration of gestation and degree of torsion. ${ }^{4}$ Authors believe with the advent of medical technology and delivery of healthcare; maternal mortality nowadays is much lower. For the past 60 years since 1960, authors found report on three maternal deaths related to uterine torsion. ${ }^{9-11}$ Apart from mortality, maternal morbidity including uterine rupture, uterine atony, sepsis, pulmonary embolism and iatrogenic complications (injury to blood vessels, urinary tract and posterior uterine wall) have been reported in the literature..$^{9,12,13}$

In the $19^{\text {th }}$ century $50 \%$ of the foetuses died as compared to $12 \%$ between $1976-1990 .{ }^{1}$ The most significant factor bearing upon foetal outcome is the degree of uterine 
torsion. The perinatal mortality was $20 \%$ for torsion less than 90 degrees and $83 \%$ for torsion more than 360 degrees. ${ }^{1}$ Referring to this case, the patient with 360 degrees uterine torsion delivered a fresh stillborn while the patient with 180 degrees torsion delivered healthy twins. Uterine venous obstruction due to uterine torsion causes increased pressure in placental cotyledons leading to abruption and fetal distress; and subsequent progress to uterine artery obstruction can cause reduction in the placental perfusion which can lead to fetal demise. ${ }^{1}$ The clinical presentation of uterine torsion is unfortunately non-specific. The most common symptom is abdominal pain however it may vary from mild discomfort to severe pain with shock, most probably neurogenic shock as illustrated in case 2. Only $11 \%$ are said to be asymptomatic 1 as in case 1 . Some patients present with abnormal fetal heart, obstructed labour, vaginal bleeding and urinary or intestinal disturbances. ${ }^{14}$ Clinical signs including twisted vaginal canal, urethral displacement and palpable round ligament are diagnostic, but rarely encountered. ${ }^{1}$

Ultrasound might detect abnormal position of ovarian vessels across the uterus or might show change in placental localization or change in position of a previously identified fibroid. ${ }^{15}$ Retrospectively in case 2, initial diagnosis of placenta previa was incorrect since after detorsion, placenta was located on the upper segment. With magnetic resonance imaging (MRI), Xshaped configuration of upper vagina could be seen instead of normal H-shaped structure. ${ }^{16}$ Nevertheless, since uterine torsion presents with non-specific symptoms or with an obstetric emergency necessitating urgent delivery, it is unlikely that imaging modalities will be used for diagnostic purposes. ${ }^{17}$

Uterine torsion may only be recognized intraoperatively. Ideally, uterus should be repositioned before proceeding with caesarean section but in many instances, this may not be possible due to advanced uterine size and limited surgical space. In such cases, deliberate transverse or vertical incision on the posterior uterine wall is required to deliver the baby. Posterior low transverse incision may cause injury to the twisted uterine/ovarian vessels and ureters but posterior vertical incision is associated with higher risk of uterine rupture although the exact risk is not known. Repositioning of the uterus is easier and may occur spontaneously once baby is delivered. In many other instances, this condition is not recognized until baby has been delivered and posterior uterine wall is inadvertently incised, as in case 1. Many authors recommend an elective caesarean section for subsequent pregnancies for patients with posterior uterine incision. Even though both hysteroscopy and laparoscopy have shown appropriate healing, the safety of vaginal birth after a posterior hysterotomy lacks substantive evidence. $^{18}$

Bilateral plication of the round ligaments has been described to prevent immediate postpartum recurrence of the torsion. ${ }^{19}$ In addition, it may help to keep the uterus in anteversion, reduce posterior uterine adhesions and future dyspareunia. ${ }^{19}$ Mustafa et al, went further to plicate the long, thin and attenuated uterosacral ligaments in their case, believing that long-term recurrence of uterine torsion could be prevented by this method. ${ }^{20}$ In view of the rarity of this condition, it is unlikely that any study could be conducted to evaluate the effectiveness of this prophylactic measure.

\section{CONCLUSION}

In conclusion, as rare as it may seem, uterine torsion which is associated with significant morbidity and mortality of mother and baby, does happen. Clinical symptoms are either absent or nonspecific, and the diagnosis is usually made intraoperatively. It is imperative to define anatomical landmarks before incising the uterus at caesarean section to avoid complications. Once diagnosed, aim of management is to restore the normal uterine anatomy and to manage the complications as a result of uterine torsion. Authors are reporting these 2 cases to increase awareness among the clinicians of this rare obstetric condition.

\section{Funding: No funding sources Conflict of interest: None declared Ethical approval: Not required}

\section{REFERENCES}

1. Jensen JG. Uterine torsion in pregnancy. Acta Obstet Gynaecol Scand. 1992;71:26-5.

2. Kawakami S, Togashi K, Sagoh T, Kimura I, Noguchi M, Takakura K, et al. Uterine deformity caused by surgery during pregnancy. J Comput Assist Tomogr. 1994;18:272-4.

3. Omurtag K, Session D, Brahma P, Matlack A, Roberts C. Horizontal uterine torsion in the setting of complete cervical and partial vaginal agenesis: a case report. Fertil Steril. 2009;91:1957.e13-5.

4. Nesbitt REL, Corner GW. Torsion of the human pregnant uterus. Obstet Gynecol Surv. 1956;11:31132.

5. Salani R, Theiler RN, Lindsay M. Uterine torsion and foetal bradycardia associated with external cephalic version. Obstet Gynaecol. 2006;108(3):8202.

6. Duplantier N, Begneaud W, Wood R, Dabezies C. Torsion of a gravid uterus associated with maternal trauma: a case report. J Reprod Med Obstet Gynecol. 2002;47(8):683-5.

7. Achanna S, Monga D, Hassan MS. Case report: torsion of a gravid horn of didelphic uterus. J Obstet Gynaecol Res. 1996;22(2):107-9.

8. Wilson D, Mahalingham A, Ross S. Third trimester uterine torsion: case report. J Obstet Gynaecol Canada. 2006;28(6):531-5.

9. Devi YL, Singh KHJ. Uterine torsion in pregnancy. J Obstet Gynaecol India. 1979;29:1260-1. 
10. Guie P, Adjobi R, N'guessan E, Anongba S, Kouakou F, Boua N, et al. Uterine torsion with maternal death: our experience and literature review. Clin Exp Obstet Gynecol. 2005;32:245-6.

11. Qureshi S, Singh U, Bansal B, Singh N. Torsion of preterm gravid uterus: a case report. Int $\mathrm{J}$ Case Reports Images. 2013;4(7):392-5.

12. Fatih FF, Gowri V, Rao K. Uterine torsion in second trimester of pregnancy followed by a successful term pregnancy. BMJ Case Rep. 2012;2012:1-3.

13. Siegler SL, Silverstein LM. Torsion of a pregnant uterus with rupture. Am J Obstet Gynecol. 1948;55(6):1053-7.

14. Bakos O, Axelsson O. Pathologic torsion of the pregnant uterus. Acta Obstet Gynaecol Scand. 1987;66:85-6.

15. Kremer JAM, van Dongen PWJ. Torsion of the pregnant uterus with a change in placental localization on ultrasound: a case report. Eur J Obstet Gynecol Reprod Biol. 1989;31:273-5.

16. Nicholson WK, Coulon CC, McCoy MC, Semelka RC. Pelvic magnetic resonance imaging in the evaluation of uterine torsion. Obstet Gynecol. 1995;85:888-90.

17. Moores KL, Wood MG, Foon RPA. Rare obstetric emergency: acute uterine torsion in a 32-week pregnancy. Case Reports. 2014;2014:2013202974.

18. Rudloff U, Joels L. Irreducible uterine torsion at caesarean section: How to deliver?. J Obstet Gynaecol. 2003;23:76-7.

19. Pelosi MA, Pelosi MA. Managing extreme uterine torsion at term. A case report. J Reprod Med. 1998;43(2):153-7.

20. Mustafa MS, Shakeel F, Sporrong B. Extreme torsion of the pregnant uterus. Aust N Z J Obstet Gynaecol. 1999;39(3):360-3.

Cite this article as: Mokhtar HN, Faridz SM, Ismail R, Yaacob N, Ramli R. Uterine torsion in pregnancy, expect the unexpected: case report. Int J Reprod Contracept Obstet Gynecol 2020;9:3473-6. 